\title{
Acellular Dermal Matrix as a Core Strut for Projection in Nipple Reconstruction: Approaches for Three Different Methods of Breast Reconstruction
}

\author{
Gui-Yong Park, Eul-Sik Yoon, Hee-Eun Cho, Byung-Il Lee, Seung-Ha Park \\ Department of Plastic Surgery and Reconstructive Surgery, Korea University College of Medicine, Seoul, Korea
}

Background The objective of this paper was to describe a novel technique for improving the maintenance of nipple projection in primary nipple reconstruction by using acellular dermal matrix as a strut in one of three different configurations, according to the method of prior breast reconstruction. The struts were designed to best fill the different types of dead spaces in nipple reconstruction depending on the breast reconstruction method.

Methods A total of 50 primary nipple reconstructions were performed between May 2012 and May 2015. The prior breast reconstruction methods were latissimus dorsi (LD) flap (28 cases), transverse rectus abdominis myocutaneous (TRAM) flap (10 cases), or tissue expander/ implant (12 cases). The nipple reconstruction technique involved the use of local flaps, including the $\mathrm{C}-\mathrm{V}$ flap or star flap. A $1 \times 2-\mathrm{cm}$ acellular dermal matrix was placed into the core with $0-$, I-, and L-shaped struts for prior LD, TRAM, and expander/implant methods, respectively. The projection of the reconstructed nipple was measured th the time of surgery and at 3,6 , and 9 months postoperatively.

Results The nine-month average maintenance of nipple projection was $73.0 \% \pm 9.67 \%$ for the LD flap group using an 0-strut, $72.0 \% \pm 11.53 \%$ for the TRAM flap group using an I-strut, and $69.0 \% \pm 10.82 \%$ for the tissue expander/implant group using an L-strut. There were no cases of infection, wound dehiscence, or flap necrosis.

Conclusions The application of an acellular dermal matrix with a different kind of strut for each of 3 breast reconstruction methods is an effective addition to current techniques for improving the maintenance of long-term projection in primary nipple reconstruction.

Keywords Acellular dermis / Nipples / Mammaplasty
Correspondence: Eul-Sik Yoon Department of Plastic Surgery, Anam Hospital, Korea University College of Medicine, 73 Inchon-ro, Seongbukgu, Seoul 02841, Korea

Tel: $+82-2-920-5368$

Fax: +82-2-922-7437

E-mail: yesanam2@korea.ac.kr

Received: 13 May 2016• Revised: 4 Sep $2016 \bullet$ Accepted: 8 Sep 2016

pISSN: 2234-6163 • elSSN: 2234-6171 • http://dx.doi.org/10.5999/aps.2016.43.5.424• Arch Plast Surg 2016;43:424-429

No potential conflict of interest relevant to this article was reported.

\section{INTRODUCTION}

Nipple reconstruction is the final surgical step in breast reconstruction. Over the years, several techniques for reconstructing the nipple following skin-sparing mastectomy and breast reconstruction have been described. Long-term maintenance of nipple projection following nipple reconstruction has been a major concern, because in most cases projection of the reconstructed 
nipple decreases as time elapses. Various "pull-out" local flaps from adjacent breast tissue such as C-V, bell, skate, and star flaps have been reported. A reported average 50\% loss of nipple projection with these techniques has been discussed in the literature [1]. Several articles have reported between $30 \%$ and $70 \%$ resorption, depending on the surgical methods used [1-5]. Recently, an acellular dermal matrix (AlloDerm, LifeCell Corp., Branchburg, NJ, USA) that can be revascularized has been used with increasing frequency for many types of breast reconstructive procedures as a supportive tissue bridge [6-16]. However, its use in primary or secondary nipple reconstruction is not well-documented. Long-term follow-up studies of nipple reconstruction with the use of an acellular dermal matrix for nipple projection are scarce. Nahabedian [17] reported the use of Alloderm, resulting in a decrease to $52 \%$ of the original height in secondary nipple reconstruction. Garramone and Lam [18] reported the use of AlloDerm in primary nipple reconstruction. The small improvements in projection shown in these results have led us to seek a modified strut technique with which to enhance long-term nipple projection. In previous studies, the insertion route of the acellular dermal matrix was uniform for all forms of prior breast reconstruction. When an autologous breast reconstruction method such as the latissimus dorsi flap or the TRAM flap is used, there is usually plenty of skin available with a thick dermal portion. However, in expander/implant-based reconstruction, there may be a paucity of skin available. Because the dead space beneath the newly reconstructed nipple has different profiles of dermal and fatty layer thickness in each of these breast reconstruction methods, we designed the tailored O, I, and $L$ struts, each with a different shape to efficiently replace the dead space in each breast mound. As the resorption rate of the reconstructed nipple may be higher when the dermal thickness of the breast skin is thinner, we believe that the use of an acellular dermal matrix to reduce resorption rates with different strut types based on the prior breast reconstruction technique shows better long-term nipple projection outcomes. The strut concept for nipple reconstruction has not been described previously in the literature. We introduce the $\mathrm{O}, \mathrm{I}$, and $\mathrm{L}$ strut concepts for the first time, each intended for a different prior reconstruction method. With this study, we were able to assess a newer technique for primary nipple reconstruction using acellular dermal matrix and to determine whether the long-term outcomes would justify its usage.

\section{METHODS}

\section{Patients}

This study retrospectively investigated nipple reconstruction cases using the acellular dermal matrix (MegaDerm, L\&C Bio Corp., Seoul, Korea) with a C-V or star flap between May 2012 and May 2015 in our center. A total of 50 primary nipple reconstructions using acellular dermal matrix were performed. The subject selection criteria were as follows: patients treated with skin-sparing mastectomy for breast cancer; 9-month or longer follow-up; no prior radiotherapy; and use of an latissimus dorsi (LD) flap, transverse rectus abdominis myocutaneous (TRAM) flap, or expander/implant for previous breast reconstruction. Exclusion criteria were as follows: secondary or tertiary nipple reconstructions and use of other nipple reconstruction techniques (skin graft, composite graft, etc.). Prior breast reconstructions were performed in 28 patients with the LD flap, in 10 patients with the free TRAM flap, and in 12 patients with the tissue expander/implant. There were no matched control groups.

\section{Surgical techniques}

Each lateral wing flap measured $2 \mathrm{~cm}$ in length, $1.5 \mathrm{~cm}$ in width, and 1.5 to $2.0 \mathrm{~cm}$ in height. Projection of the nipple was usually 1.5 to $2.0 \mathrm{~cm}$ at completion, based on the projection of the opposite nipple. The operations were performed under local anesthesia using 1:3 lidocaine:saline solution without epinephrine. The senior author selected a C-V flap or star flap pattern according to the tension of the skin envelope intraoperatively. When the central wing donor site had to be undermined to achieve a tension release, we used a star flap design. The dermal flap was outlined, incised, and elevated in the subcutaneous plane with a scalpel. The donor sites of the lateral wings were undermined to minimize the tension around the new nipple and closed with 4-0 Vicryl (Ethicon Inc., Somerville, NJ, USA) and 5-0 nylon. The bilateral elevated wing flaps were wrapped around each other and sutured with 6-0 nylon.

A $1 \times 2-\mathrm{cm}$ precut of Megaderm was inserted and sutured with 5-0 Vicryl around the pocket created by the approximated lateral wing flaps. The position of the acellular dermal matrix was determined by the characteristics of the dead space according to the method used in prior breast reconstruction to serve as a central strut to support the projection and prevent nipple drooping. In the LD flap, there is a thick layer of dermis and a thin layer of subcutaneous fat. To support the projection and fill the dead space mainly from the lower fat portion of the mound, we positioned the $\mathrm{O}$-shaped strut flat beneath the nipple base under the lateral wings of the local flap (Fig. 1). In the TRAM flap mound, there was less dermal thickness, but a more sufficient portion of fat than in the LD flap group. The I-shaped strut was inserted vertically, wrapped between the lateral wings of the local flap to support the dermal portion of the reconstructed nipple. Finally, in the expander/implant group, there was a thinner layer of der- 


\section{Fig. 1. Core struts with each breast reconstruction method}

Primary nipple reconstruction using acellular dermal matrix as a central strut is shown. (A) The schematic illustration shows that the position of the acellular dermal matrix was determined by the method of prior breast reconstruction to serve as a central strut to support the projection. he 0-strut for an LD flap (a), I-strut for TRAM flap (b), and L-strut for expander/implant (c) are shown. The 3 cases demonstrate the relative differences in dermal and fat thickness. (B) A piece of $1 \times 2 \mathrm{~cm}$ MegaDerm is trimmed into an ' 0 ' shape for insertion and fixed with 5-0 Vicryl as the 0 -strut transversely beneath the wrapped bilateral flaps in cases following LD flap reconstruction. (C) MegaDerm is placed into the core between the lateral wing flaps vertically in the I-strut in cases following TRAM flap breast reconstruction. (D) MegaDerm is inserted as the L-strut from the space between the lateral wings to the space beneath the new nipple following expander/implant breast reconstruction. The bottom of the 'L' is positioned flat on the base of the new nipple and is hidden in this photo. The column of the 'L' shown in the photo is fixed vertically and wrapped inside the lateral wings. LD, latissimus dorsi; TRAM, transverse rectus abdominis myocutaneous.

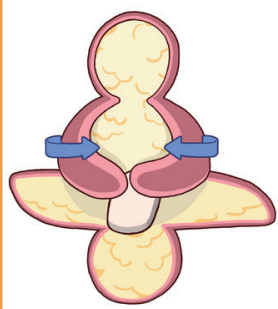

a

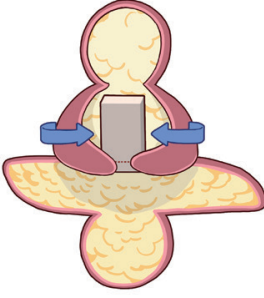

b

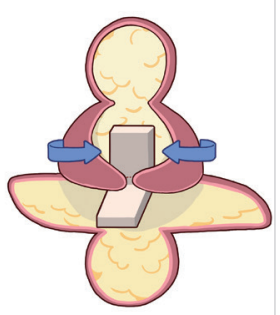

C
(A)

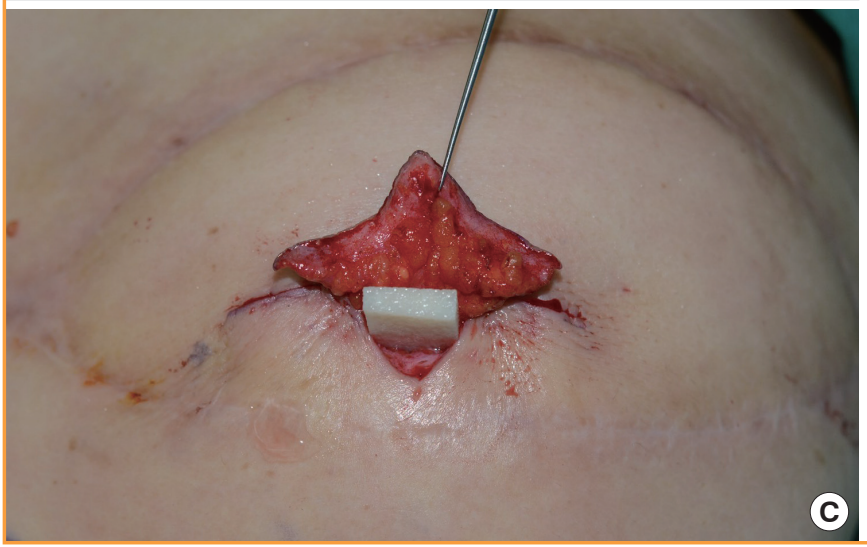

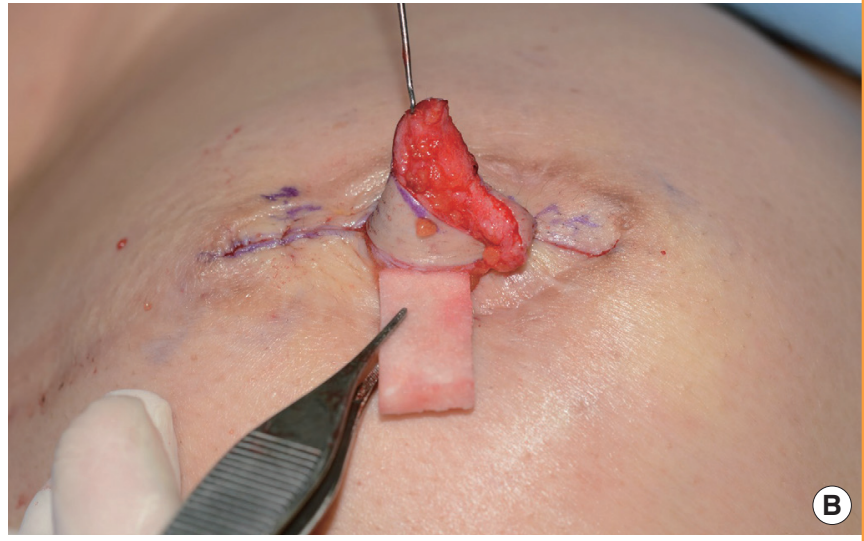

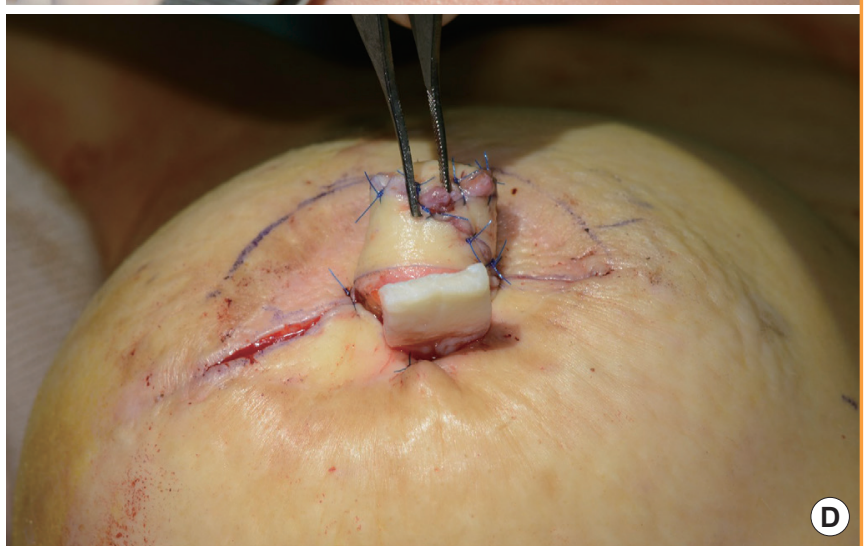

mis and fat in the reconstructed nipple and the surrounding donor tissue than with the other two methods. The L-shaped strut was constructed by hatching and folding the straight acellular dermal matrix and positioned from the base to the tip of the reconstructed nipple to support the dermal and fat portion simultaneously.

The top of the new nipple was then closed using 6-0 nylon. After the incisions were closed, nitric oxide ointment (Rectogesic) was applied to aid flap circulation and molded foam dressings were used to preserve nipple projection. Postoperative use of this dressing was maintained for 6 weeks.

\section{Assessment of projection}

The projection of the reconstructed nipple was measured in millimeters with calipers from the base of the nipple to the tip of the nipple at the time of surgery and at 3, 6, and 9 months post- operatively. Maintenance of nipple projection was calculated as the percentage of final projection measurement relative to the initial projection measurement. Surgical records and complications were also reviewed.

\section{RESULTS}

The mean age of the patients was 49 years (range, 37 to 66 years). The follow-up period ranged from 9 months to 17 months (mean, 11 months). The ratio of the number of patients receiving the C-V flap to those receiving the star flap was 7:3. In the LD flap group using the $\mathrm{O}$-strut, nipple projection fell from an average of $1.6 \mathrm{~cm}$ at the time of surgery to an average of $1.1 \mathrm{~cm}$ at 9 months. In the TRAM flap group using an I-strut, nipple projection measurements fell from an average of $1.5 \mathrm{~cm}$ at the time of surgery, to an average of $1.1 \mathrm{~cm}$ at 9 months. For the tissue expander/ 


\section{Fig. 2. Clinical photos in an LD flap patient}

A lateral view of a 41 -year-old female breast demonstrating nipple projection at postoperative 6 days (A) and the 9-month (B) followup, after LD flap reconstruction. An 0 -strut was applied flat underneath the new nipple in this LD case. The projection of the nipple was $1.7 \mathrm{~cm}$ on postoperative day 1 and $1.2 \mathrm{~cm}$ at postoperative 9 months. LD, latissimus dorsi.

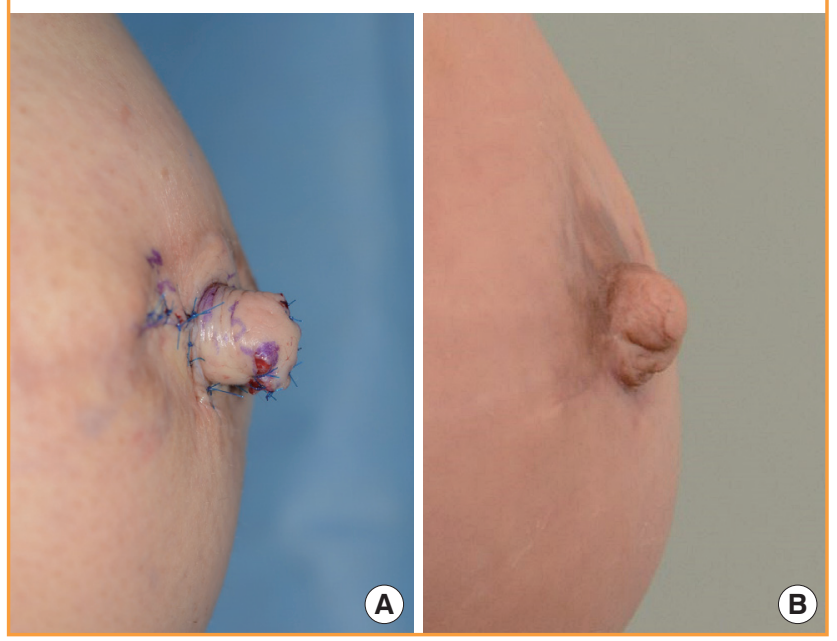

\section{Fig. 3. Clinical photos in a TRAM flap patient}

A lateral view of a 47-year-old female breast demonstrating nipple projection of $1.6 \mathrm{~cm}$ immediate postoperatively $(\mathrm{A})$ and $1.1 \mathrm{~cm}$ at the 9-month (B) follow-up, after TRAM flap reconstruction. The I-strut was positioned vertically and wrapped between the lateral wings of the C-V flap. TRAM, transverse rectus abdominis myocutaneous.
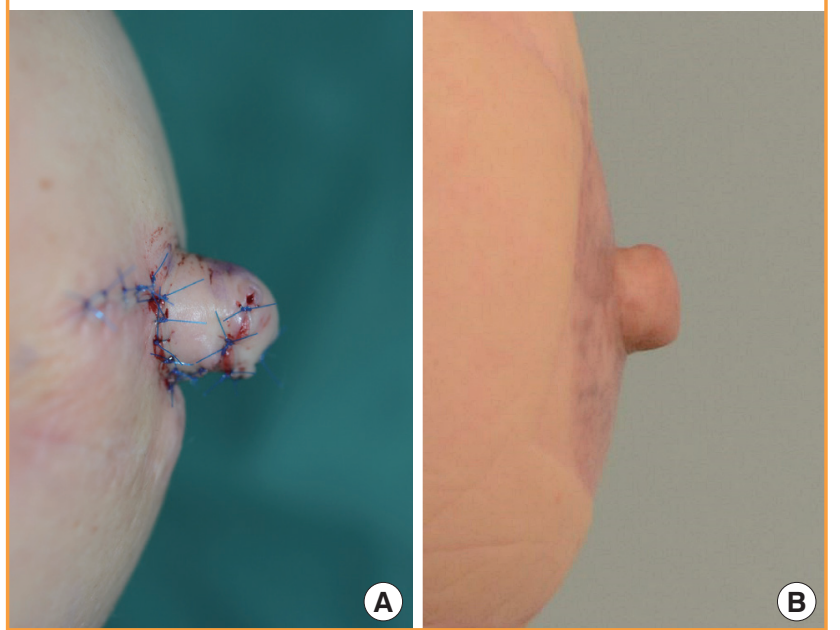

implant group using an L-strut, nipple projection fell from an average of $1.5 \mathrm{~cm}$ at the time of surgery, to an average of $1.0 \mathrm{~cm}$ at 9 months postoperatively. The average 9 -month maintenance of nipple projection was $73.0 \% \pm 9.67 \%$ (mean, standard deviation) for the LD flap group using the O-strut, $72.0 \% \pm 11.53 \%$ for the TRAM flap group using the I-strut, and 69.0\% $\pm 10.82 \%$ for the tissue expander/implant group using the L-strut. Postoperative photographs of each reconstruction are shown in Figs. $2-4$. The mean operative time was 32 minutes. The average time

\section{Fig. 4. Clinical photos in a tissue expander/implant patient}

A lateral view of a 59-year-old female breast demonstrating nipple projection of $1.5 \mathrm{~cm}$ immediate postoperatively $(\mathrm{A})$ and $1.1 \mathrm{~cm}$ at the 9-month (B) follow-up, after tissue expander/implant reconstruction. The L-strut was applied at the base and in the space between the lateral wings of the reconstructed nipple.
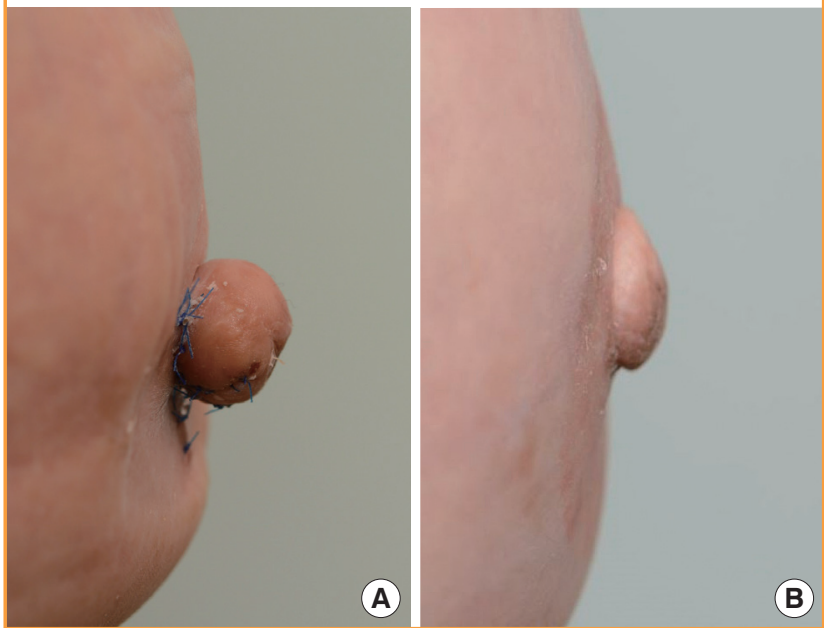

to complete healing was 3 weeks. There were no cases of flap necrosis, infection, wound dehiscence, or any other associated complications.

\section{DISCUSSION}

Long-term maintenance of nipple projection in nipple reconstruction is a major concern because projection of the reconstructed nipple decreases as time elapses in most cases. The reason for this postoperative projection loss is multifactorial. We believe that poor circulation, skin contractures related to skin tension, flap necrosis, and dead space beneath the flap or a thinner dermal layer may promote the absorption process. The ratio of epidermis, dermis, and subcutaneous fat varies and is dependent on the quality of skin, the type of breast reconstruction, and the surgical technique used [17]. LD flaps are most likely to be related to increased dermal thickness of the skin and a small amount of subcutaneous fat. TRAM flaps are associated with a thin dermal layer and a larger volume of subcutaneous fat. Following tissue expander/implant reconstruction, the breast dermis and subcutaneous fat are much thinner than those following autologous flap reconstruction because the breast tissue has been expanded. Thinner dermal and subcutaneous fat layers may lead to increased dead space, contraction, central depression, and consequent shrinkage or drooping of the reconstructed nipple. Moreover, the amount of dead space beneath the reconstructed nipple originating from the varying ratios of dermis and subcutaneous fat in each method of breast reconstruction 
may account for the projection loss or nipple drooping as the dead space contracts.

Recently, acellular dermal matrix has become readily available in a precut packaged format. This can be easily applied between the elevated dermal flaps for improved nipple reconstruction. Nahabedian [17] was the first to describe the use of AlloDerm augmentation in secondary and tertiary nipple reconstruction using an elongated $\mathrm{C}$ flap and a C-V flap. This study involved 8 patients with both implant and autologous tissue reconstructed breasts. Garramone and Lam [18] reported that the 12-month average maintenance of nipple projection was $56 \%$ for a TRAM flap group and $47 \%$ for a tissue expander group with Alloderm use. The use of acellular dermal matrix as a core strut in primary nipple reconstruction has been demonstrated in our study. Although shrinkage of the nipple did occur, the final projection observed at 9 months was greater than that of the other reported 12-month follow-up study (73.0\% for the LD flap group, $72.0 \%$ for the TRAM flap group, and $69.0 \%$ for the tissue expander/ implant group versus $56 \%$ for the previous TRAM flap group and $47 \%$ for the tissue-expanded group [18]). These results were encouraging because we supposed our 1-year follow-up results would not be much different from the 9-month follow-up results according to previous studies reporting the stabilization of the nipple projection at 6 months postoperatively $[1,19]$. We presume that the acellular dermal matrix fills the dead space beneath the nipple and the supporting tissue caused by lower dermal or subcutaneous fat thickness in each breast reconstruction, thus alleviating the contracture and loss of the projection. Further biopsy-proven studies should investigate how tissue incorporation is observed in the host tissue where the acellular dermal matrix was originally placed and further follow-up observations must be followed.

In previous studies, the insertion pattern of the acellular dermal matrix was uniform whatever the method used for the prior breast reconstruction. When the $\mathrm{LD}$ flap is used, there is plenty of skin available, with a thick dermal portion and thin subcutaneous fat. In the TRAM flap, there is little skin available, with a thin dermal portion and thick subcutaneous fat. In expander/ implant-based reconstruction, there may be a paucity of skin and subcutaneous fat available because of expansion of the skin envelope. Differences in both the dermal thickness and the amount of subcutaneous fat with each method of breast reconstruction may result in different amounts of dead space beneath the local flap. If not filled, this dead space can result in nipple contraction and drooping. Given that the resorption rate of the reconstructed nipple would increase with decreased skin and subcutaneous fat thickness and increased dead space, we have developed a strut application that varies according to breast reconstruction method. This study focused on addressing the different characteristics of the dead space according to the prior reconstruction method. We assumed that filling the dead space in each different breast mound with an appropriately tailored volume and shape of acellular dermal matrix would reduce the contraction forces and thus the extent of absorption of the reconstructed nipple. The LD flap comprises a thick layer of dermis and a thin layer of subcutaneous fat. To support the projection and fill the dead space mainly from the lower fat portion of the mound, we used the O-strut transversely beneath the nipple in the LD flap mound (Fig. 1A). In the TRAM flap mound, the dermis was thinner, but with a greater portion of fat than in the LD flap group. The I-strut was thus applied vertically and wrapped with the flap to support the dermal portion of the reconstructed nipple. Finally, in the expander/implant group, there was a lower volume of both dermis and fat in the reconstructed nipple and the surrounding donor tissue relative to the other two methods. In this group, we positioned the L-strut to support the dermal and fat portions simultaneously. Each of these strut shapes are intended to contribute to reducing the long-term resorption rate as an efficient dermal and fatty replacement.

In our study there are a few limitations. First, the TRAM flap and expander/implant groups contained only a small number of patients. A further study including a larger number of patients should be performed. Second, the follow-up period in our study was relatively short, given that similar studies have reported 1-year follow-up results. However, we chose 9 months postoperatively as our endpoint because in a prior study of our center, we found that the nipple projection showed little change between the 9-month follow-up and 1-year follow-up [19]. Shestak et al. [1] also reported that the greatest loss of projection occurred in the first 3 months and stabilized at 6 months with a skate or C-V flap. We thus believe a 9-month postoperative period can be considered acceptable for reporting results as "long-term". Although follow-up beyond 12 months and biopsy-proven dermal replacement are still needed as follow-ups to our study, the present study has successfully demonstrated the viability of the concept of using different strut types to maintain nipple projection in nipple reconstruction after different breast reconstruction methods. Applying acellular dermal matrix in one of three different shaped struts for each method of breast reconstruction is an effective addition to the current techniques for improving the maintenance of long-term projection in primary nipple reconstruction.

\section{REFERENCES}

1. Shestak KC, Gabriel A, Landecker A, et al. Assessment of 
long-term nipple projection: a comparison of three techniques. Plast Reconstr Surg 2002;110:780-6.

2. Kroll SS, Reece GP, Miller MJ, et al. Comparison of nipple projection with the modified double-opposing tab and star flaps. Plast Reconstr Surg 1997;99:1602-5.

3. Lee PK, Lim JH, Ahn ST, et al. Nipple reconstruction with dermis(scar tissue) graft and C-V flap. J Korean Soc Plast Reconstr Surg 2006;33:101-6.

4. Ahn HC, Choi EK, Hwang WJ. Nipple reconstruction using various local flaps. J Korean Soc Plast Reconstr Surg 2003; 30:183-8.

5. Kroll SS. Nipple reconstruction with the double-opposing tab flap. Plast Reconstr Surg 1999;104:511-4.

6. Cothren CC, Gallego K, Anderson ED, et al. Chest wall reconstruction with acellular dermal matrix (AlloDerm) and a latissimus muscle flap. Plast Reconstr Surg 2004;114:1015-7.

7. Baxter RA. Intracapsular allogenic dermal grafts for breast implant-related problems. Plast Reconstr Surg 2003;112: 1692-6.

8. Salzberg CA. Nonexpansive immediate breast reconstruction using human acellular tissue matrix graft (AlloDerm). Ann Plast Surg 2006;57:1-5.

9. Gamboa-Bobadilla GM. Implant breast reconstruction using acellular dermal matrix. Ann Plast Surg 2006;56:22-5.

10. Zienowicz RJ, Karacaoglu E. Implant-based breast reconstruction with allograft. Plast Reconstr Surg 2007;120:37381.
11. Breuing KH, Colwell AS. Inferolateral AlloDerm hammock for implant coverage in breast reconstruction. Ann Plast Surg 2007;59:250-5.

12. Preminger BA, McCarthy CM, Hu QY, et al. The influence of AlloDerm on expander dynamics and complications in the setting of immediate tissue expander/implant reconstruction: a matched-cohort study. Ann Plast Surg 2008;60:510-3.

13. Spear SL, Parikh PM, Reisin E, et al. Acellular dermis-assisted breast reconstruction. Aesthetic Plast Surg 2008;32:41825.

14. Nahabedian MY. AlloDerm performance in the setting of prosthetic breast surgery, infection, and irradiation. Plast Reconstr Surg 2009;124:1743-53.

15. Maxwell GP, Gabriel A. Use of the acellular dermal matrix in revisionary aesthetic breast surgery. Aesthet Surg J 2009; 29:485-93.

16. Duncan DI. Correction of implant rippling using allograft dermis. Aesthet Surg J 2001;21:81-4.

17. Nahabedian MY. Secondary nipple reconstruction using local flaps and AlloDerm. Plast Reconstr Surg 2005;115:205661.

18. Garramone CE, Lam B. Use of AlloDerm in primary nipple reconstruction to improve long-term nipple projection. Plast Reconstr Surg 2007;119:1663-8.

19. Kim DY, Dhong ES, Yoon ES, et al. Long-term result of nipple reconstruction using skate flap after breast reconstruction. J Korean Soc Plast Reconstr Surg 2011;38:401-7. 